\title{
Post-training consumption of wheat gluten hydrolysate suppresses the delayed onset of muscle injury in soccer players
}

\author{
KAZUHIRO AOKI ${ }^{1}$, YOSHIMITSU KOHMURA ${ }^{1}$, YOSHIO SUZUKI ${ }^{2}$, NATSUE KOIKAWA ${ }^{1}$, \\ MASAFUMI YOSHIMURA ${ }^{2}$, YUKIHIRO AOBA ${ }^{1}$, NORIFUMI FUKUSHI ${ }^{1}$, \\ KEISHOKU SAKURABA ${ }^{2}$, ISAO NAGAOKA ${ }^{3}$ and KEISUKE SAWAKI ${ }^{1}$ \\ ${ }^{1}$ School of Health and Sports Science; ${ }^{2}$ Graduate School of Health and Sports Science, \\ Juntendo University, Inzai, Chiba; ${ }^{3}$ Department of Host Defense and Biochemical Research, \\ Juntendo University Graduate School of Medicine, Tokyo, Japan
}

Received February 24, 2012; Accepted March 20, 2012

DOI: $10.3892 /$ etm.2012.539

\begin{abstract}
Wheat gluten hydrolysate (WGH) is reported to suppress the muscle injuries associated with exercise in long distance running and weight training. In the present study, we investigated the effects of WGH consumption on suppression of muscle injury after soccer training in a double-blind crossover study. Immediately after a mini soccer game, six soccer players consumed $18.0 \mathrm{~g}$ of $\mathrm{WGH}$, and muscle injury was investigated using serum creatine kinase $(\mathrm{CK})$ as an indicator. The results showed a significant increase in serum CK from immediately after exercise to $12 \mathrm{~h}$ after exercise stress $(\mathrm{p}<0.05)$ in the placebo group, while serum CK decreased during this same time period in the WGH group, and the difference between the two groups was significant. This suggests that WGH consumption suppresses delayed-onset muscle injury after exercise in soccer.
\end{abstract}

\section{Introduction}

In various competitive sports, the importance of recovery in training has become evident, and nutrition is considered to play a major role. In recent years, supplements such as amino acids have been used after training.

Wheat gluten hydrolysate (WGH), one of these supplements, has attracted attention. WGH is a peptide prepared by enzymatic treatment of wheat gluten; it has excellent dispersibility and dissolution properties and is used as a glutamine-enriching ingredient in enteral nutrients (for what is known as immunonutrition) that are used perioperatively (1).

Correspondence to: Mr. Kazuhuro Aoki, Department of Sports Science, School of Health and Sports Science, Juntendo University, 1-1 Hiraga-gakuendai, Inzai, Chiba 270-1695, Japan

E-mail:k.aoki@sakura.juntendo.ac.jp

Key words: wheat gluten hydrolysate, muscle injury, soccer, football
In the field of sports, investigations have been conducted on the effects of WGH on endurance exercise (2-4) and weight training (4,5). Koikawa et al reported that post-exercise consumption of WGH suppressed subsequent muscle injury, as indicated by serum creatine kinase (CK) activity (2) and the effect was reproduced in a larger randomized control trial (3). Similar results were also obtained by pre-ingestion of WGH in weight training (5).

On the other hand, there have been no studies concerning its effects on training in ball games, which involves more complex movement. Among ball games, soccer involves complex instantaneous movements that are repeatedly performed. In the present study, we report on our investigation of the effects of WGH consumption on muscle injury from soccer training in soccer players in a double-blind crossover study.

\section{Materials and methods}

Subjects. Eight male soccer players belonging to a college soccer club were enrolled in the study. They had a history of competitive athletics since high school and were capable in college-level competition. They were given thorough explanation of the objectives, methods and ethical considerations of the study and provided written consent for participation. After completion of the exercise protocols, two subjects were excluded from the data analyses since one presented with an elevated activity in serum creatine kinase $>1000 \mathrm{U} / 1$ due to a possible slight distortion and the other subject was affected by a upper respiratory infection. Characteristics of the remaining six subjects are documented in Table I.

Study supplement. The test supplement in the form of a granulated powder containing $6.0 \mathrm{~g}$ of WGH per packet was used. Other ingredients included anhydrous glucose, anhydrous citric acid, sweetener (sucralose), fragrance (lemon micron) and thickener (pullulan). The placebo contained water soluble fiber $(6.0 \mathrm{~g})$ instead of WGH, with other ingredients being the same. Supplements were packaged in aluminum packets sealed on three sides, each containing $9 \mathrm{~g}$ per packet. Test and 
Table I. Characteristics of the subjects.

\begin{tabular}{lccccccc}
\hline & \multicolumn{7}{c}{ Subjects } \\
\cline { 2 - 6 } Characteristics & A & B & C & D & E & F & Mean \pm SD \\
\hline Age (years) & 19 & 20 & 19 & 19 & 19 & 19 & $19.2 \pm 0.4$ \\
Height (cm) & 173.0 & 181.0 & 179.0 & 175.0 & 172.0 & 169.0 & $174.8 \pm 4.49$ \\
Body weight $(\mathrm{kg})_{\text {Soccer position }^{\mathrm{a}}}$ & 62.0 & 76.0 & 73.0 & 75.0 & 71.0 & 65.0 & $70.3 \pm 5.65$ \\
History of competition (years) & $\mathrm{MF}$ & $\mathrm{FW}$ & $\mathrm{MF}$ & $\mathrm{MF}$ & $\mathrm{DF}$ & $\mathrm{MF}$ & \\
& 10 & 14 & 13 & 13 & 13 & 11 & $12.3 \pm 1.5$ \\
\hline
\end{tabular}

${ }^{\mathrm{a}} \mathrm{MF}$, midfielder; FW, forward; DF, defense.

Table II. Composition of the supplement (per 9-g packet).

\begin{tabular}{lll}
\hline Supplement & \multicolumn{1}{c}{ Ingredient } & Content $(\mathrm{g})$ \\
\hline Test & Wheat gluten hydrolysate & 6 \\
& Anhydrous glucose & 2 \\
& Anhydrous citric acid & 0.9 \\
& Sucralose (as sweetener) & 0.016 \\
& Lemon flavor & 0.084 \\
Placebo & Pullulan (as thickener) & 0.135 \\
& Polydextrose & 6 \\
& Anhydrous glucose & 2 \\
& Anhydrous citric acid & 0.9 \\
& Sucralose (as sweetener) & 0.016 \\
& Lemon flavor & 0.084 \\
& Pullulan (as thickener) & 0.135 \\
\hline
\end{tabular}

placebo supplements were prepared by Nisshin Pharma, Inc. (Tokyo, Japan). The detailed ingredients are listed in Table II.

Study design. A double-blind crossover method was used in this study, with the subjects randomized into two groups. For the first round of the study, subjects were divided into one group who consumed WGH and another group who consumed the placebo ( 3 subjects in the WGH group and 3 subjects in the placebo group). In the second round, study supplements were alternated (3 subjects in the WGH group and 3 subjects in the placebo group).

Under the direction of the team coach, subjects trained four-on-four on half a soccer field (43 $\mathrm{m}$ x $28.5 \mathrm{~m}$ ) by playing six sets of 5-min mini-games. There was a 3-min rest between each set. Immediately after exercise stress, subjects consumed either 3 packets of test or placebo supplement. In addition, health and dietary surveys were conducted for the period starting one day before until the day after exercise stress. Subjects completed the health survey, which was in question and answer form and included items such as physical subjective condition, body weight, body temperature and bowel movements. For the dietary survey, subjects answered questions on the content and quantity of food consumed, and took photographs; nutritional intake was calculated based on this information.

Blood samples were taken at rest before exercise stress, immediately after exercise stress, and at $12 \mathrm{~h}$ after exercise stress and serum creatine kinase (CK), lactate dehydrogenase (LDH), aspartate aminotransferase (AST), alanine transaminase (ALT) and white blood cell count (WBC) levels were measured. SRL (Tokyo) conducted the analyses.

A one-week washout period was established at the end of the first round of experiments. The supplements consumed by the subjects were switched and the second round of experiments was conducted. During the study period, with the exception of the exercise stress established by the study, subjects were forbidden from high intensity training associated with muscle injury. This study was reviewed and approved by the Ethics Committee of the Juntendo University School of Health and Sports Science.

Statistical analysis. Following ANOVA, Dunnett's method was used to compare data before exercise, immediately after exercise and at $12 \mathrm{~h}$ after exercise, with the pre-exercise data used as a control. A paired t-test was used to compare corrected CK levels (with post-exercise levels expressed as 100) with levels at $12 \mathrm{~h}$ after exercise in the WGH and placebo groups. The risk ratio was set at $<5 \%$. SPSS ver. 19.0 (IBM, Chicago, IL) was used to analyze the data.

\section{Results}

The results of the health, training and dietary surveys during the study period showed no significant differences between the WGH and placebo groups, and there were no results that had an effect on the study findings (data not shown).

Alterations in blood parameters prior to and after exercise are summarized in Table III. Significant increases in serum $\mathrm{CK}$ and $\mathrm{LDH}$ were observed in both groups after exercise when compared with pre-exercise levels. In the control group, the serum CK level was significantly higher post exercise than the level prior to exercise, and the CK level was even higher at $12 \mathrm{~h}$ after exercise. When CK activity was adjusted by the level after exercise expressed as 100 , a significant $(\mathrm{p}<0.05)$ difference was observed between the groups at $12 \mathrm{~h}$ after exercise; these levels were $95.0 \pm 14.9$ and $116.2 \pm 13.9$ in the WGH and placebo groups, respectively (Fig. 1). On the other hand, LDH, 
Table III. Alterations in the blood activity prior to and post soccer exercise training.

\begin{tabular}{|c|c|c|c|c|c|}
\hline Group & CK (U/l) & $\operatorname{AST}(\mathrm{U} / \mathrm{l})$ & $\operatorname{ALT}(\mathrm{U} / \mathrm{l})$ & LDH (U/l) & $\operatorname{WBC}\left(10^{3} / \mu 1\right)$ \\
\hline \multicolumn{6}{|l|}{ WGH } \\
\hline Pre-exercise & $284.3 \pm 51.8^{\mathrm{a}}$ & $22.5 \pm 2.4^{\mathrm{b}}$ & $19.5 \pm 2.4$ & $191.5 \pm 22.1^{\mathrm{c}}$ & $5.7 \pm 1.0$ \\
\hline Post-exercise & $362.0 \pm 37.0$ & $26.8 \pm 2.6$ & $21.2 \pm 2.4$ & $235.0 \pm 21.4$ & $7.6 \pm 2.4$ \\
\hline $12 \mathrm{~h}$ after exercise & $343.3 \pm 56.3$ & $23.5 \pm 2.8$ & $18.3 \pm 3.0$ & $206.3 \pm 11.5$ & $6.1 \pm 1.0$ \\
\hline \multicolumn{6}{|l|}{ Control } \\
\hline Pre-exercise & $177.5 \pm 35.1^{\mathrm{c}, \mathrm{d}}$ & $20.2 \pm 2.0$ & $17.7 \pm 4.8$ & $178.3 \pm 14.3^{\mathrm{c}}$ & $6.1 \pm 0.9$ \\
\hline Post-exercise & $249.5 \pm 30.5$ & $24.0 \pm 2.3$ & $19.0 \pm 4.6$ & $224.2 \pm 16.3$ & $8.2 \pm 1.5$ \\
\hline $12 \mathrm{~h}$ after exercise & $287.8 \pm 33.2$ & $18.0 \pm 4.0$ & $17.2 \pm 3.7$ & $188.2 \pm 10.5$ & $6.2 \pm 0.7$ \\
\hline
\end{tabular}

CK, creatine kinase; AST, aspartate aminotransferase; ALT, alanine transaminase; WBC, white blood cell count. ${ }^{\mathrm{a}} \mathrm{p}<0.05 \mathrm{vs} .12 \mathrm{~h}$ after exercise; ${ }^{\mathrm{b}} \mathrm{p}<0.05$ vs. post-exercise; ${ }^{\mathrm{c}} \mathrm{p}<0.01$ vs. post-exercise; ${ }^{\mathrm{d}} \mathrm{p}<0.01$ vs. $12 \mathrm{~h}$ after exercise.

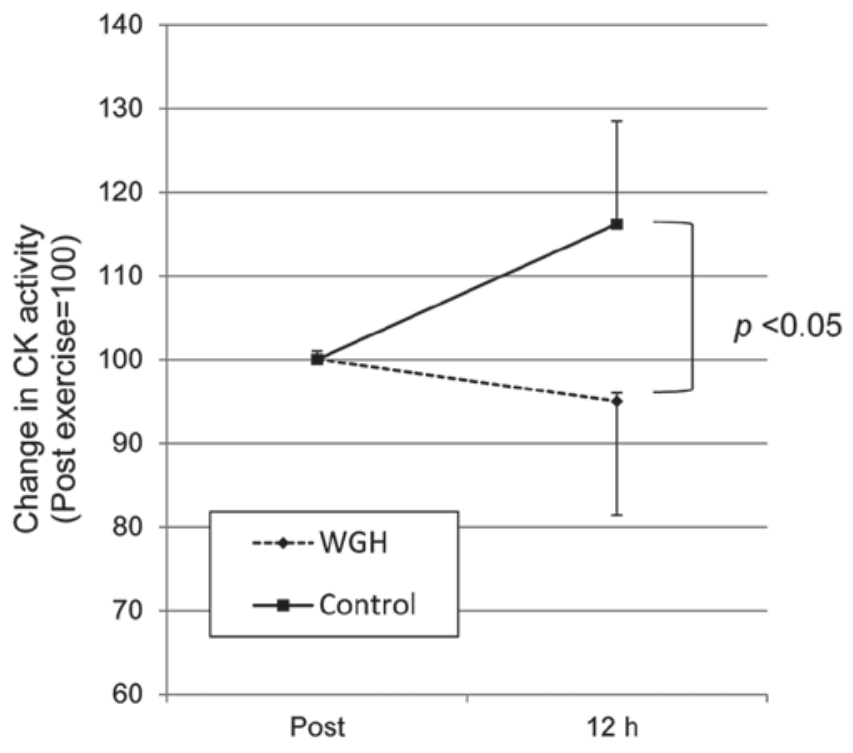

Figure 1. Post-exercise change in serum CK activity, normalized by postexercise activity expressed as 100 .

ALT and AST activities returned to pre-exercise levels at $12 \mathrm{~h}$ after exercise with no differences between groups. WBC count was elevated after exercise and returned to pre-exercise levels in both groups. No correlation was found in post-exercise WBC levels and CK activities after $12 \mathrm{~h}$.

\section{Discussion}

Soccer is characterized by repeated sprints and stops, and the endurance to run for an entire 90-min game is also important. The soccer training employed in this study took place on half a soccer field, and consisted of repeated sprints and stops conducted based on adequate instructions by the team coach. This type of soccer training is similar to an actual game format. Previously, we investigated the suppressive effects of WGH on muscle injury due to endurance exercise (2-4) including half-marathons (3) and from strength training (5) using elevation of CK activity as an indicator. In the present study, we investigated the effect on exercise stress from ball games. After mini-games, CK and LDH levels were higher than pre-exercise levels, thus confirming that muscle injury had occurred. On the other hand, there were no distinct increases in ALT or AST levels. AST is mainly present in cardiac muscle, liver, skeletal muscle and the kidneys, while ALT is present in the liver, followed by kidneys and other tissues. Therefore, injury resulting from mini-game activities would be less evident than with $\mathrm{CK}$ and $\mathrm{LDH}$, which exist in abundance in skeletal muscle. Consequently, it is clear that muscle injury due to the mini-game was not sufficiently severe to show a significant difference in ALT or AST levels.

Serum CK is known to peak 24-48 h after exercise stress, which is due to delayed-onset muscle injury accompanied by inflammation (5). The fact that CK levels were higher at $12 \mathrm{~h}$ after exercise than immediately after exercise in the control group confirms that delayed-onset muscle injury also occurred in the present study. On the other hand, in the WGH group, $12 \mathrm{~h}$ after exercise, the mean levels were lower than they were immediately after exercise, at a level at which there were no statistically significant differences with pre-exercise levels. These results are in accordance with those in investigation of distance running (2-4) and showed that consumption of WGH after exercise stress suppresses subsequent delayedonset muscle injury. In fact, when CK levels were normalized by values after exercise stress expressed as 100, there was a significant difference in comparison with the control group.

Inflammation plays a role in the post-exercise increase in CK activity. Paulsen et al reported a positive correlation bewteen post-exercise leukocyte count and CK activity (6). Koikawa et al suggested that the post-exercise WBC count could predict the peak CK activity after exercise (2). In contrast, in the present study, the WBC count did not show a significant correlation. The WBC count after the mini-game increased but the levels were within the reference range (Table III). This could be the reason that the correlation was not apparent.

Ball games such as soccer frequently involve a series of competitions, including league games and post-games, and post-training recovery is crucial for improving competitive power. As a result, post-exercise consumption of WGH may be useful as it enables quick recovery. 
However, the present study only investigated serum CK kinetics in one training session, and it is necessary to investigate the kinetics over a series of training sessions. Additionally, in the present study, muscle injury was evaluated based on serum CK dynamics, but to evaluate recovery or overcompensation, evaluation from a more functional perspective such as muscle strength, is also necessary.

In conclusion, consumption of WGH after soccer training inhibited the release of CK into the blood for $12 \mathrm{~h}$ after exercise stress. Thus, consumption of WGH after exercise appears to suppress subsequent delayed-onset muscle injury and to accelerate recovery.

\section{Acknowledgements}

This study was supported, in part, by Nisshin Pharma, Inc., which was the manufacturer of the wheat gluten hydrolysate supplement used in this study. A section of this study was presented at the 18th Annual Meeting of the Japanese Society of Clinical Medicine in 2007.

\section{References}

1. Sawaki K, Takaoka I, Sakuraba K and Suzuki Y: Effect of distance running and subsequent intake of glutamine rich peptide on biomedical parameters of male Japanese athletes. Nutr Res 24: 59-71, 2004.

2. Koikawa N, Nakamura A, Nagaoka I, Aoki K, Sawaki K and Suzuki Y: Delayed-onset muscle injury and its modification by wheat gluten hydrolysate. Nutrition 25: 493-498, 2009.

3. Hirao T, Koikawa N, Aoki K, Sakuraba K, Shimmura Y, Suzuki Y and Sawaki K: Female distance runners show a different response to post-workout consumption of wheat gluten hydrolysate compared to their male counterparts. Exp Ther Med 3: 641-644, 2012. doi 10.3892/etm.2012.446.

4. Takanashi Y, Suzuki Y, Aoki K, Koikawa N, Shimmura Y, Sakuraba K and Kaneko K: Effect of an intake of WGH on strength training for a thrower's. Jpn J Clin Sports Med (In press).

5. Armstrong RB: Mechanisms of exercise-induced delayed onset muscular soreness: a brief review. Med Sci Sports Exerc 16: 529-538, 1984.

6. Paulsen G, Benestad HB, Strøm-Gundersen I, Mørkrid L, Lappegård KT and Raastad T: Delayed leukocytosis and cytokine response to high-force eccentric exercise. Med Sci Sports Exerc 37: 1877-1883, 2005. 